\title{
Non-random distribution of spermatogonia in rats: evidence of niches in the seminiferous tubules
}

\author{
H. Chiarini-Garcia ${ }^{1}$, A. M. Raymer ${ }^{2}$ and L. D. Russell ${ }^{2 \dagger}$ \\ ${ }^{1}$ Laboratory of Cellular Biology, Department of Morphology, Federal University of Minas Gerais, \\ Belo Horizonte, MG 31270-901, Brazil; and ${ }^{2}$ Department of Physiology, School of Medicine, \\ Southern Illinois University, Carbondale, IL 62901, USA
}

The relationships and distribution of spermatogonia were studied as a function of the stage of the seminiferous epithelium cycle in rats. Primitive spermatogonia in the mouse are located along regions of the basal lamina that face the interstitium. Before studying the distribution of spermatogonia in rats, it was necessary to characterize the various types of spermatogonia, as recently performed for mice. The Strauss' linear index (Li) selectivity method was then used and spermatogonia of the $A_{\text {single }}\left(A_{s}\right)$ to $A_{\text {aligned }}$ $\left(A_{a l}\right)$ lineage were preferentially found to be located in regions opposing the interstitium at stages $\mathrm{V}, \mathrm{VII}$ and IX of the spermatogenic cycle. Because relatively little tubuleto-tubule contact occurs in rats, the aim of this study was to determine whether tubule-to-tubule contact or tubule proximity (or alternatively, the amount of interstitium) was an important factor in spermatogonial position. In this regard, another method (tubule proximity) was devised to determine spermatogonial position that accounted for the presence of adjacent tubules. This method showed that the position of tubules, rather than tubule contact, was more accurate than the Li method in determining the location of spermatogonia in the rat. The results also showed a non-random distribution of spermatogonia resembling that of the mouse, and that tubule-to-tubule contact is not essential for the positioning of spermatogonia. In conclusion, the results of this study strongly indicate that the most primitive type $A$ spermatogonia $\left(A_{s}, A_{\text {paired }}\right.$ and $\left.A_{a l}\right)$ in rats are present in niches located in those areas of the seminiferous tubules that border the interstitial tissue.

\section{Introduction}

For an organ to function properly there must be a topographical relationship between its cells. For example, the skin of mammals is distributed evenly over the entire body except for regions where functional specialization occurs. The regulation of skin topography during normal function and after injury implies that the stem cells should be positioned in such a manner that their progenitors give rise to a layer of skin that has a relatively uniform distribution of progenitor cells. The concept of stem cell topography or stem cell niche is readily understood when it applies to the integument (Miller et al., 1997), intestine (Bjerkness and Cheng, 1999) and haematopoietic tissue (Spangrude et al., 1988).

With respect to the testis which is also a self-renewing cell system, it is obvious that there must also be some regulation of stem cell topography in mammals. Spermatogenesis occurs within seminiferous tubules and the spermatozoa are released into the lumen of the tubule. The tubules produce spermatozoa around the seminiferous tubule in a uniform manner. Even after

${ }^{\dagger}$ Deceased

Email: chiarini@icb.ufmg.br destruction of much of the testis by irradiation (van den Aardweg et al., 1982; Meistrich, 1998) or after transplantation of germ cells (Parreira et al., 1998; Brinster, 2002) or after vitamin A replacement in vitamin A-deficient rodents (van Pelt and de Rooij, 1990a,b), normal uniform and symmetrical spermatogenesis resumes in the testis.

It has been shown that the stem cell function is probably controlled by the neighbouring differentiated cells and their cellular substrates. The microenvironments that control the stem cell self-renewal and progeny production in vivo are known as niches. However, as it is difficult to identify individual stem cells, the niches have remained a theoretical construct. Nevertheless, with identification of the niches it will be possible to manipulate the stem cells and their surroundings and, in this way, perhaps to discover how they are regulated (Spradling et al., 2001).

Chiarini-Garcia et al. (2001) have shown that spermatogenesis in the mouse is uniform and that primitive spermatogonia are distributed in a non-random manner. At certain stages, the majority of primitive spermatogonia, the $A_{\text {single, }}, A_{\text {paired }}$ and $A_{\text {aligned }}$ cells $\left(A_{s}, A_{p r}\right.$ and $A_{a l}$, respectively), are located along the tubule wall in regions that oppose the interstitium. Spermatogonia are thus spaced in locations where they are apart from 
each other and initiate spermatogenesis at evenly distributed loci. Their spacing is thus a key regulator of the uniform production of spermatozoa. Regions in which mouse seminiferous tubules abut each other show few primitive spermatogonia. Thus, it was hypothesized that specific factors emanating from the interstitium cause primitive spermatogonia to occupy a niche along the interstitium. Alternatively, the contact of seminiferous tubules may result in factors that lead to the repulsion of spermatogonia from these regions.

Tubule-to-tubule contact is extensive in the mouse. Three to seven seminiferous tubules in mice are shown to have extensive contact with one another when the tissue is viewed under ideal fixation conditions. There is less tubule-to-tubule contact in rats than in mice. The rat testes can be readily perfused and the distribution of its primitive spermatogonia characterized, as performed for the mouse (Chiarini-Garcia and Russell, 2001, 2002). The aim of this study was to determine whether the pattern of regulation of spermatogenesis distribution that occurs in mice occurs in rats too.

\section{Materials and Methods}

Animals, tissue collection and processing

The testes of four Sprague-Dawley rats aged 160 days were perfusion-fixed with $5 \%(\mathrm{w} / \mathrm{v})$ glutaraldehyde (biological grade) in cacodylate buffer $\left(0.05 \mathrm{~mol} \mathrm{I}^{-1}\right.$; $\mathrm{pH}$ 7.4) according to the method of Sprando (1990). The mean testis mass was $1.63 \pm 0.07 \mathrm{~g}(n=8)$. Small slabs of testes were post-fixed in an osmium:ferrocyanide mixture (Russell and Burguet, 1977), dehydrated in ethanol, infiltrated with propylene oxide and embedded in Araldite 502 (Electron Microscopy Sciences, Fort Washington, PA). Tissues were embedded so that crosssections of seminiferous tubules were obtained when samples were cut into sections ( $1 \mu \mathrm{m}$ in thickness) for light microscopy. Over 18 light microscope slides from different regions of each testis (from each animal) were made. The maintenance of the animals was in full compliance with the standard laboratory animal care protocols approved by Institutional Animal Care and Use Committee (IACUC).

\section{Determining types of spermatogonia}

The stages of the spermatogenic cycle were determined using the map provided by Russell et al. (1990), as slightly modified from the staging scheme of Leblond and Clermont (1952). In the XIV stages of the seminiferous epithelium cycle, all type A spermatogonia were photographed with a Nikon Optiphot Microscope (Nikon Inc., Garden City, NJ). Over 100 digital images of type A spermatogonia were photographed at each stage. Images were adjusted for resolution (600 dots per inch), sharpness $(180 \%$ with radius set at 6.0 pixels and threshold set at 0) and contrast/gray level (sigmoidal curve) using Photoshop 6.0 (Adobe Systems, Inc., Mountain View, CA). For each stage of the epithelial cycle, images of spermatogonia were grouped according to those possessing similar morphological characteristics.

\section{Determining the position of spermatogonia}

Two methods were used to study the position of spermatogonia: tubule-to-tubule contact and tubule-totubule proximity. For these purposes, tubules at stages II and III, V, VII, X and XII of the spermatogenic cycle were selected on the basis of the presence of primitive and differentiated type A spermatogonia. A camera lucida was used to draw the boundaries (peritubular tissue) of the tubules at $\times 240$ magnification and at the stages cited above. For each stage selected, nine tubules were drawn.

\section{Tubule-to-tubule contact}

The tubule perimeter was divided into three different regions (Fig. 1). The portion of the tubule in which the peritubular tissue contacted other tubules was known as tubule-to-tubule contact. A contact was defined as the absence of visible space between the peritubular tissues of adjacent seminiferous tubules. The interface of the peritubular tissue with the interstitial space was known as the interstitial region. Another region was designated as the intermediate region, in which the interstitial region and the tubule-to-tubule contact region joined. This region constituted the space between tubuleto-tubule contact and peritubular tissue, beginning where the tubules were in close contact up to the point where they diverged at the width of two thicknesses of the peritubular tissue. In the rat, there were two to four regions in which each tubule related to the interstitium, although the range was zero to six.

A digitizer was used to determine the total length of each region. The total length of each region was averaged from nine tubules analysed from each animal and the percentage of length of each region was calculated. The profile of the various types of type A spermatogonia were outlined using the camera lucida and the positions of type A spermatogonia were recorded as being opposite the interstitial, intermediate or tubule-to-tubule contact areas. The total number of cells in each animal was determined for each of these three positions. The mean number of type A spermatogonia in each of three regions and for the selected stages was estimated and the percentage value obtained.

Strauss's linear selectivity index (Linear Index, Li) (Strauss, 1979) was used to analyse the region selection by type $\mathrm{A}$ spermatogonia in the basal compartment of seminiferous tubules of the rats. This method was applied by Chiarini-Garcia et al. (2001) to study the distribution of type A spermatogonia in mice. The Li index values $(\mathrm{Li})$ 

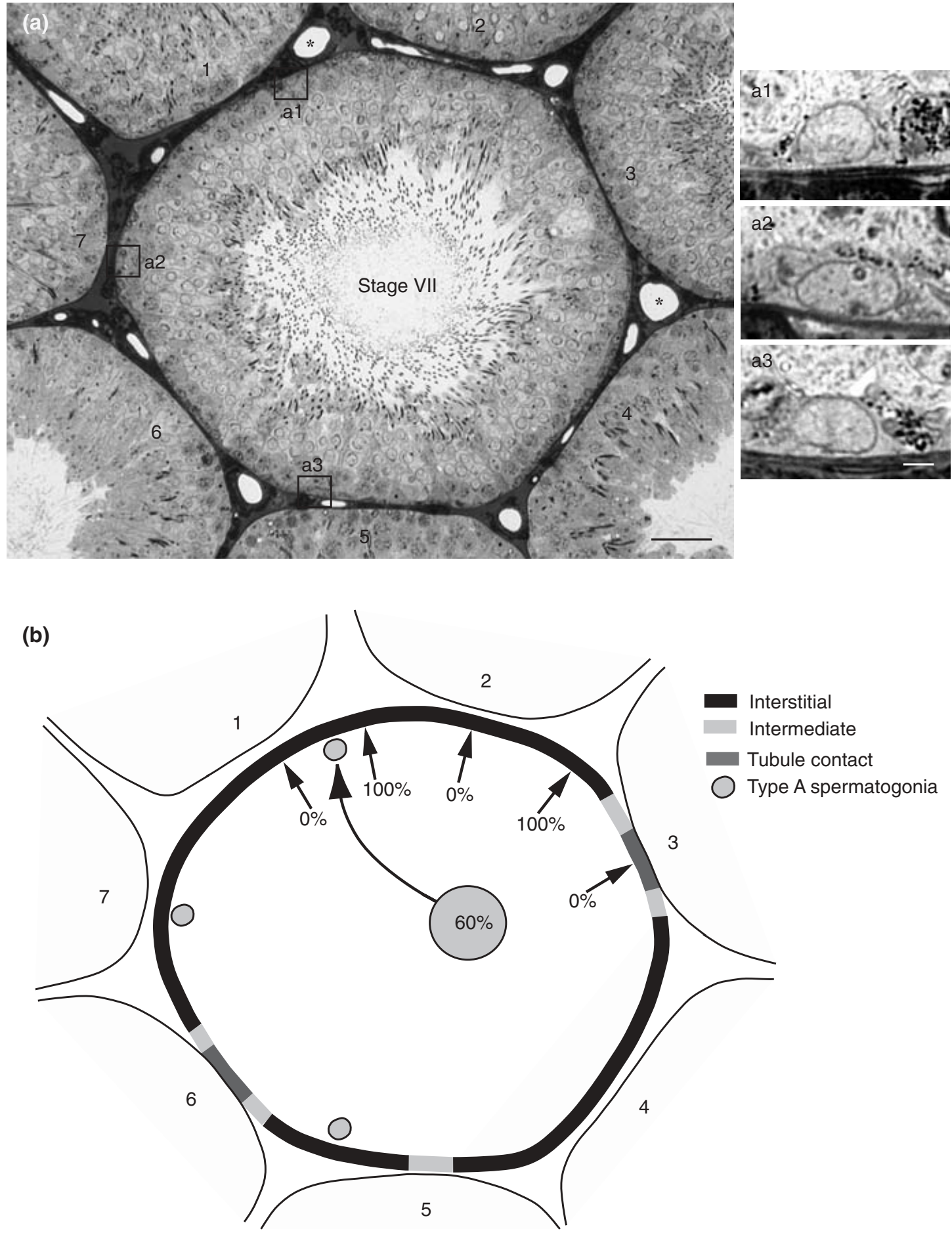

Fig. 1. The scheme used to determine the position of rat spermatogonia based on their tubule-to-tubule contact and tubule proximity to other tubules. (a) A light micrograph of a stage VII tubule is shown in close proximity to seven other tubules. However, only two of the tubules (3 and 6) make contact with the stage VII tubule $(\times 350$ magnification). The spermatogonia found in the tubule are shown to the right (a1, a2 and a3) ( $\times 1300$ magnification). Asterisks represent blood vessels. (b) Drawing of the tubule in (a). For the tubule-to-tubule contact method, the spermatogonia were outlined on the three distinct regions of the seminiferous tubule: interstitium, intermediate and tubule contact. When the tubule proximity method is applied, for instance, the spermatogonium at the top of the illustration lies $60 \%$ of the way between a point at which an adjacent seminiferous tubule is closest to the tubule containing the spermatogonia of interest and the mid-point of the interstitium. Scale bars represent (a) $30 \mu \mathrm{m}$ and (a1-a3) $3.6 \mu \mathrm{m}$. 
were calculated by the formula:

$$
L i=r_{i}-p_{i}
$$

where $r_{i}$ is the proportion of spermatogonia in a specific region and $p_{i}$ the proportion of length in the same region.

The estimated sampling variance $s^{2}$ of $L i$ is

$$
s^{2}=\frac{r_{i}\left(1-r_{i}\right)}{n_{r}}+\frac{p_{i}\left(1-p_{i}\right)}{n_{p}}
$$

where $n_{r}$ is the total number of spermatogonia in a specific region and $n_{p}$ is the total length of the same region.

$L i$ values were calculated for each tubular region, and resulted in $L i$ values ranging from -1 to 1 with zero representing a random position of type A spermatogonia. Positive numbers represented positive selection (preference) of the given region, whereas negative numbers represented negative selection (avoidance) of the given region. $\mathrm{Li}$ values were used to plot a graph with $95 \%$ confidence intervals. A $t$ test was used to determine whether $L i$ values were significantly different from zero (random), and also for stage comparison among the regions in a single location (with $n_{r}+n_{p}-2$ degrees of freedom; $P<0.05)$. ANOVA was performed to show homogeneity of the animals with respect to spermatogonial distribution and comparisons indicated no differences in $L i$ values.

\section{Tubule-to-tubule proximity}

As there were few areas of tubule-to-tubule contact, a second method was used to determine the position of spermatogonia. For this method, two points were marked on the tubule containing the spermatogonium. The first point was the region considered as the central interstitium; the second was the point at which the nearest adjoining tubule most closely approximated the tubule containing the spermatogonium. The first point was considered as $100 \%$ and the second point as $0 \%$. Thus, the spermatogonia could be considered along a distance that ranged from 0 to $100 \%$. The closer the spermatogonia to the interstitium, the higher the percentage recorded (Fig. 1).

A percentage for each spermatogonium was recorded at each stage and a mean for all spermatogonia was determined after showing virtually no differences among animals using ANOVA. Graphs, herein termed tubule proximity graphs, were plotted as a function of stage. These graphs were prepared by dividing the mean percentage of spermatogonia by $50 \%$, the percentage expected if the distribution were random. When the ratio calculated is 1 , the type A spermatogonia distribution is random; a ratio of $>1$ indicates preference for the interstitial region and a ratio of $<1$ indicates avoidance. The ratios determined were compared longitudinally (stage-wise comparisons) using a $t$ test $(P<0.05)$ and were compared within stages using chi-squared test to determine whether they were significantly different from random $(P<0.05)$.

\section{Results}

The tissues were fixed well so that there were no spaces between tubules that were considered to be artifacts, and no shrinkage artifact within the tubule was evident. Types of rat spermatogonia were similar to those described for the mouse (Chiarini-Garcia and Russell, 2001), although they were not as distinct as in the mouse. Similar grouping of cells could be demonstrated. In all stages of the spermatogenic cycle, $A_{s}, A_{p r}$ and $A_{a l}$ cells were found to have a mottled nucleus, with one subtype showing a substantially more mottled nucleus (Fig. 2). Criteria were also presented for identifying $A_{1}, A_{2}, A_{3}, A_{4}$, intermediate type and type $B$ spermatogonia.

A comparison of several types of spermatogonial cell is shown when such types of cell lie next to each other within the seminiferous tubule (Fig. 3). A yes or no decision tree is also presented which aids in discrimination of the type of cell (Fig. 4).

Seminiferous tubules of the rat made contact with up to six other seminiferous tubules. Only $0.43 \pm$ $0.49 \%$ (SEM) of tubules were not contacting other tubules; $6.08 \pm 4.07 \%$ contacted one tubule; $19.67 \pm$ $4.36 \%$ contacted two tubules; $39.68 \pm 2.19 \%$ contacted three tubules; $23.90 \pm 6.36 \%$ contacted four tubules; $8.71 \pm 3.95 \%$ contacted five tubules and $1.53 \pm 0.51 \%$ contacted six tubules.

The mean percentage of the seminiferous tubule regions free of contact with other seminiferous tubules, known as the interstitial area, was $68.50 \pm 1.82 \%$; the mean percentage of seminiferous tubule regions considered intermediate was $13.86 \pm 1.52 \%$; the mean percentage of tubule-to-tubule contact was $17.64 \pm 0.71 \%$.

A linear index graph applying the formula from the ratios obtained for all types of spermatogonia $\left(A_{S}\right.$ to $\left.A_{3}\right)$ (Fig. 5a) shows that there are significant differences from random in spermatogonial distribution in combined stage II and III up to stage XII. There were no significant differences between the distributions when the comparison was made longitudinally as a function of stage.

The Li graph showing only the most primitive spermatogonia $\left(A_{s}, A_{p r}\right.$ and $\left.A_{a l}\right)$ indicates that all cells of this type are significantly different from random, preferring the interstitial regions and avoiding both the intermediate and tubule-to-tubule contact regions (Fig. 5b). The $\mathrm{Li}$ graph of the more advanced spermatogonia $\left(A_{1}-A_{3}\right)$ shows them to be initially random $\left(A_{1}\right.$ spermatogonia at stage $\mathrm{VII})$, and then shows that their progeny $\left(\mathrm{A}_{2}\right.$ spermatogonia at stage IX and $\mathrm{A}_{3}$ spermatogonia at stage XII) have a significant preference for the interstitium (Fig. 5C).

The tubule proximity graph of the distribution of all spermatogonia indicates that the group prefers the 
(a)
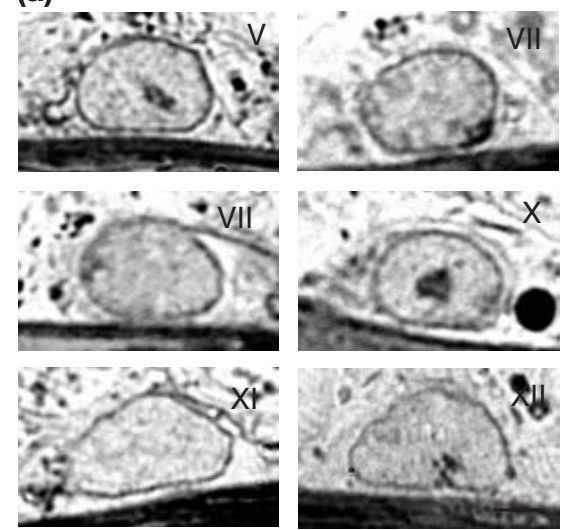

(b)
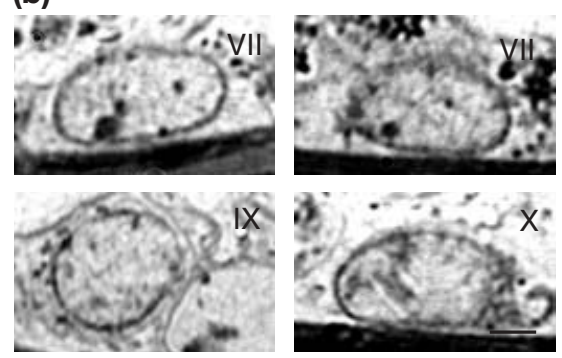

(c)
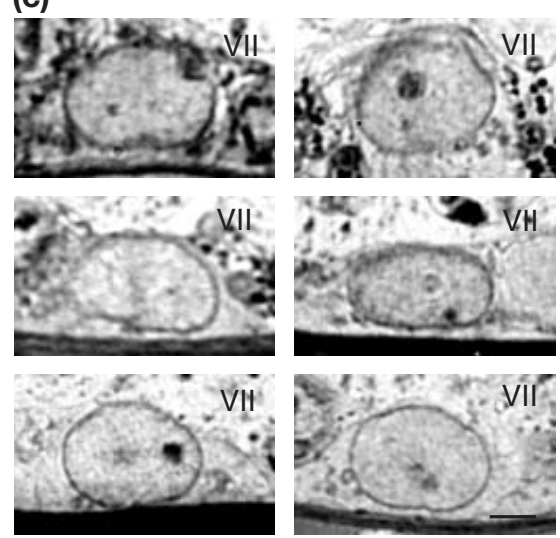

(d)
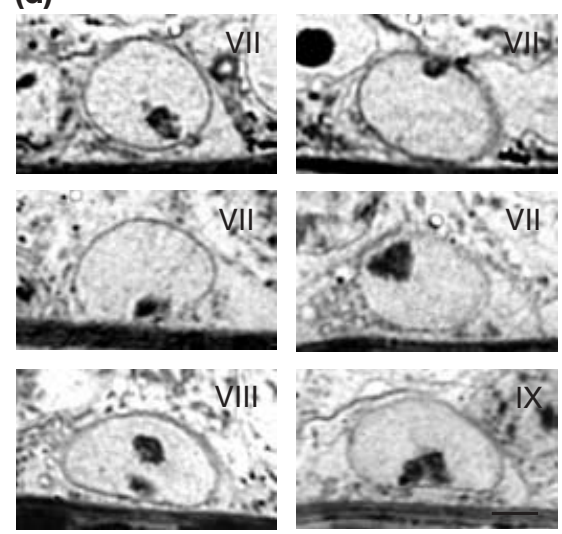

$A_{s}, A_{p r}$ and $A_{a l}$

Cytoplasm density - low to moderate density.

Nuclear shape - oval.

Nucleolus - up to one in section, low density.

Heterochromatin-splotchy chromatin of low density throughout the nucleus contrasting with intervening regions of euchromatin (mottled nucleus); rare areas of dense heterochromatin along the nuclear envelope.

Euchromatin - contributes to the mottled appearance.

Major distinguishing feature from predecessor - see $A_{a l}$ subtypes which have more pronounced mottling.

Major distinguishing feature from the sucessor cell - the nucleus in $A_{s}-A_{a l}$ cells is mottled and not finely granular. The nucleolus is considerably less prominent.

\section{$A_{\text {al }}$ subtype}

Cytoplasm density - sometimes dense.

Nuclear shape - oval or somewhat flattened.

Nucleolus - up to one in section.

Heterochromatin - mottled (more so than other cells).

Euchromatin - pronounced clear areas between mottled heterochromatin

Major distinguishing feature from $A_{a l}$ cells - there is a greater extent of nuclear mottling compared with other $A_{a l}$ cells.

\section{$A_{\text {al }}$ to $A_{1}$ (transition cell)}

Cytoplasm density-low to moderate density.

Nuclear shape - oval.

Nucleolus - one or two small nucleoli in section.

Heterochromatin - low density finely mottled throughout nucleus.

Euchromatin - very small light regions between heterochromatin.

Major distinguishing feature from predecessor cell - lesser extent of nuclear mottling compared with $\mathrm{A}_{\mathrm{al}}$.

Major dintinguishing feature from sucessor cell-greater extent of mottling of the nuclear heterochromatin than $\mathrm{A}_{1}$.

$A_{1}$

Cytoplasm density - low to moderate.

Nuclear shape - oval.

Nucleolus - up to two nucleoli in each section; well circumscribed, darkly stained, often finely reticulated, usually peripherally.

Heterochromatin - not detected due to almost complete absence of mottling.

Euchromatin - not detected along nuclear envelope. Mottling almost absent.

Major distinguishing features from predecessor cell - texture of nucleus is more finely granular than transition cells.

Major distinguishing features from sucessor cell - virtually no heterochromatin along the nuclear envelope.

Fig. 2. Continued on page 674 . 
(e)

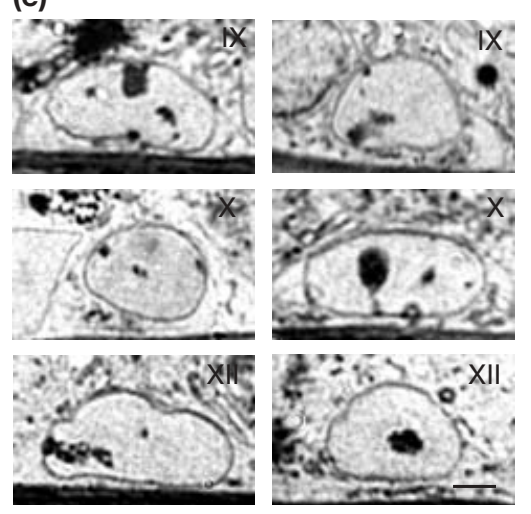

(f)

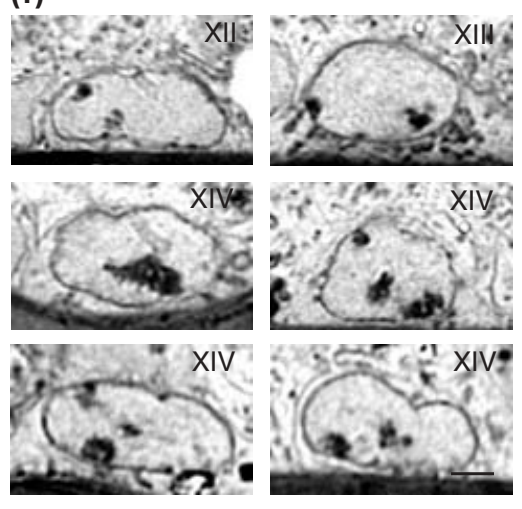

(g)

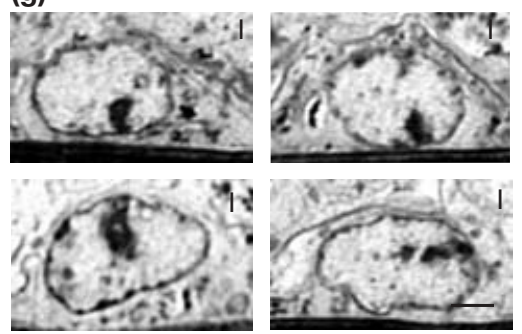

(h)

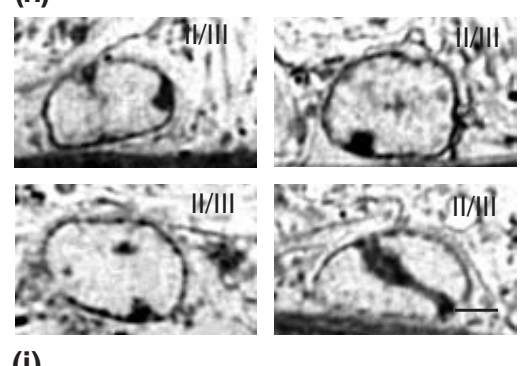

(i)

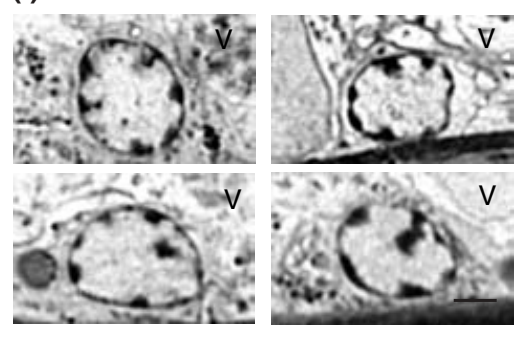

$\mathrm{A}_{2}$

Cytoplasm density - low to moderate density.

Nuclear shape - oval, similar to $A$.

Nucleolus - sometimes less compact, more stringy than $A_{1}$.

Heterochromatin - flecks of heterochromatin along the nuclear envelope occupying less than $10 \%$ of the nuclear envelope.

Euchromatin - slight indication of fine mottling of the nucleus. Mottling does not approach the extent of that described for $A_{\text {al }}$ cells.

Major distinguishing feature from predecessor cell - presence of small chromatin flecks along the nuclear envelope; presence of less compact, but stringy, nucleolus.

Major distinguishing feature from sucessor cell - less heterochromatin lining the nuclear envelope.

\section{$\mathrm{A}_{3}$}

Cytoplasm density - low to moderate density.

Nuclear shape - oval.

Nucleolus - large, stringy, reticulated and extends deeply into the nucleus; up to three nucleoli visualized in section.

Heterochromatin - about $15 \%$ of the nuclear envelope is lined with heterochromatin.

Euchromatin - slight increase in the extent of the mottling of the nucleus.

Major distinguishing feature from predecessor cell - increase in heterochromatin

lining the nuclear envelope.

Major distinguishing feature from sucessor cell - less heterochromatin lining nucleus.

\section{$A_{4}$}

Cytoplasm density - low to moderate density.

Nuclear shape - oval to slightly round in section.

Nucleolus - sometimes the nucleolus may bridge across the nucleus.

Heterochromatin - chromatin lines from $20-70 \%$ of the sectioned nucleus.

Euchromatin - similar to $\mathrm{A}_{3}$ cells.

Distinguishing feature from predecessor cell - more heterochromatin lining nucleus.

Distinguishing feature from sucessor cell - considerably less heterochromatin rimming the nucleus.

\section{Intermediate type}

Cytoplasm density - low to moderate density.

Nuclear shape - oval to round.

Nucleolus - one or two, compact nucleoli.

Heterochromatin $-70-100 \%$ of the nucleus rimmed by heterochromatin, heterochromatin is shallow in regions and flaky in other regions.

Euchromatin - clear areas are slightly larger than $\mathrm{A}_{4}$ cells.

Major distinguishing feature from predecessor cell - increase in chromatin rimming the nucleus.

Major distinguishing feature from sucessor cell - heterochromatin rimming nucleus is both shallow and flaky compared with the compact rounded heterochromatin of type B cells.

\section{Type B}

Cytoplasm density - low to moderate density.

Nuclear shape - round to slightly oval in section.

Nucleolus - small, compact and darkly stained; usually one per section.

Heterochromatin - rounded heterochromatin clumps located periodically along the nuclear membrane occupying 50 to $70 \%$.

Euchromatin - mottling due to contrast between heterochromatin and euchromatin.

Major distinguishing feature from predecessor cell - balls of dense heterochromatin rim a portion of the nucleus.

Fig. 2. The various types of rat spermatogonia accompanied by detailed descriptions of their features at different stages of the spermatogenic cycle ( $\times 1300$ magnification). (a) $A_{\text {single, }} A_{\text {paired }}$ and $A_{\text {aligned }}$, (b) $A_{\text {aligned }}$ subtype, (c) $A_{\text {aligned }}$ to $A_{1}$, (d) $A_{1}$, (e) $A_{2}$, (f) $A_{3}$, (g) $A_{4}$, (h) intermediate type and (i) type B. Scale bar represents $3.6 \mu \mathrm{m}$. 
regions nearest to the interstitium only at stages $\mathrm{V}$ and VII, whereas the rest of the stages are not significantly different from random (Fig. 6a). Considering only the primitive spermatogonia $\left(A_{s}, A_{p r}\right.$ and $\left.A_{a l}\right)$ significant differences from random were observed in stages $V$, $\mathrm{VII}$ and $\mathrm{XI}$ (Fig. 6b). Among only the more advanced spermatogonia $\left(A_{1}-A_{3}\right)$, only those at stage VII $\left(A_{1}\right)$ significantly preferred the interstitial location (Fig. 6C).

\section{Discussion}

In the present study the different types of spermatogonia in rats were characterized providing the essential foundation for examining the distribution of spermatogonia in a species other than the mouse, in which non-random distribution of spermatogonia was first described by Chiarini-Garcia et al. (2001). The results of this part of the study showed that in rats as in mice spermatogonia are preferentially located near the interstitial regions. Secondly, in rats, which have fewer tubule-to-tubule contacts than mice it was found that tubule-to-tubule contact is less accurate in determining the spermatogonial niche than is the tubule proximity.

The ability to distinguish types of spermatogonia was based on characteristics similar, but not identical, to those in the mouse (Chiarini-Garcia and Russell, 2001). The most primitive cells $\left(A_{s}\right.$ to $\left.A_{a l}\right)$ were present at all stages of the cycle of the seminiferous epithelium. The nuclei of these cells were mottled, consisting of regions of heterochromatin and euchromatin. Even a subtype (possibly $A_{s}$ or $A_{p r}$ ) was identified on the basis of the low frequency of cells seen and the extremely pronounced mottling of the chromatin. $A_{1}$ cells demonstrated finely and evenly distributed chromatin, whereas in $A_{2}, A_{3}$, $\mathrm{A}_{4}$ and intermediate types of spermatogonia there was a progressive increase in the amount of heterochromatin rimming the nuclear envelope. Type B spermatogonia had fewer chromatin rimming the nucleus than its predecessor cells, but the density and shape of the chromatin was distinctly different from them. This accurate morphological characterization of spermatogonia in rats may be useful in future investigations to categorize spermatogonia without using the stage of the spermatogenic cycle as the primary criterion, either in whole-mounted seminiferous tubules or sectioned material. It should be emphasized that the results of the present study are applicable only if the tissues are well fixed and prepared in the manner described. For example, even if section thickness is increased, the amount of heterochromatin rimming the nucleus will appear greater. Even under the conditions specified herein, some of the types of cell, such as $A_{2}$ and $A_{3}$ spermatogonia, have a very similar appearance, and several cells must be examined to make a distinction between them.

Tubule-to-tubule contact, as seen in the mouse and to a lesser extent in the rat, is characteristic of many
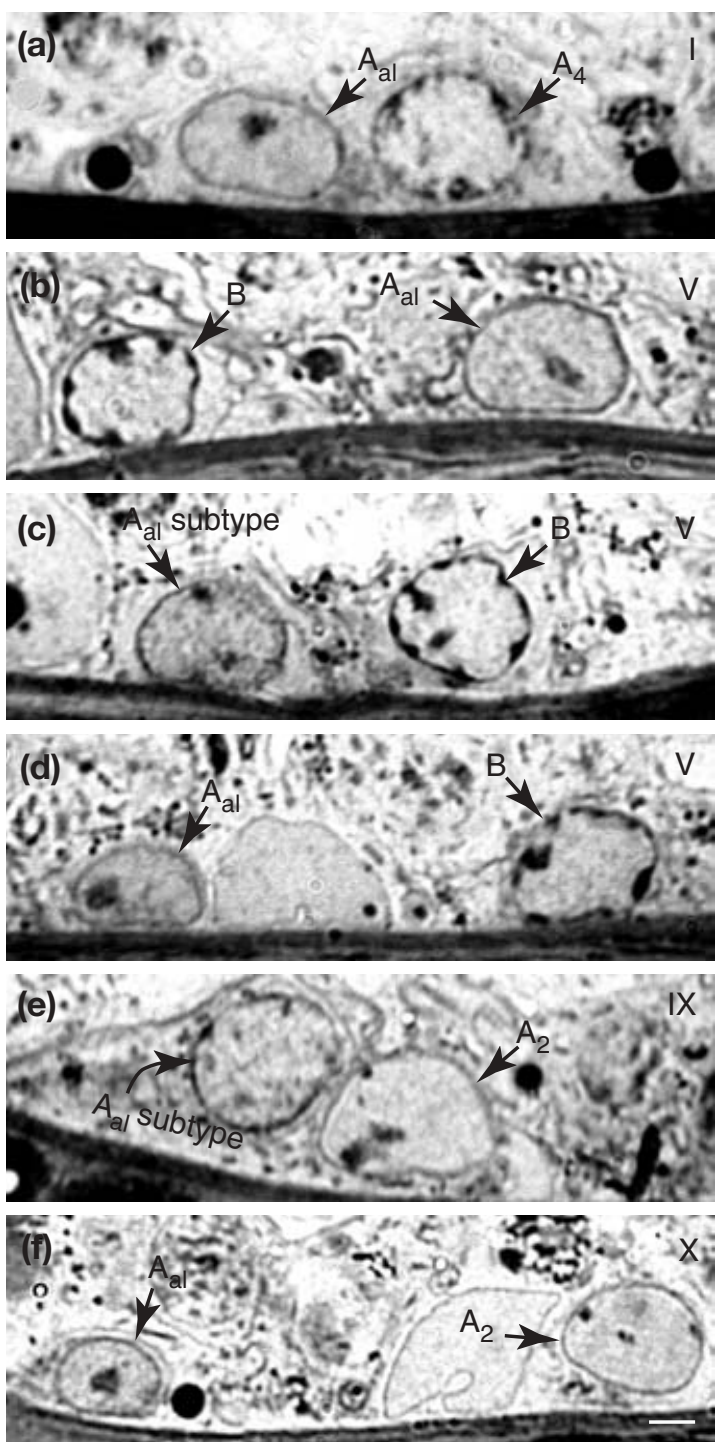

Fig. 3. (a-f) Different types of rat spermatogonia $\left(A_{\text {aligned }}, A_{\text {aligned }}\right.$ subtype, $A_{3}, A_{4}$ and type B) at stages (a) I, (b-d) V, (e) IX and (f) $X$ of the spermatogenic cycle ( $\times 1300$ magnification). Scale bar represents $3.6 \mu \mathrm{m}$.

species. So far species in which there is such extensive interstitial tissue that no tubule-to-tubule contact occurs have not been examined. The data obtained from the present study allowed determination of whether tubule contact per se or proximity to the tubules is important for spermatogonial position. The Li method, using the three regions originally designated (tubule-to-tubule, intermediate and interstitial), does not take into account the situation in which tubules may be close but do not make contact, as is common in the mouse. By close, we mean that the approximation of the tubules in the rat may qualify as interstitial in the mouse. Thus, if proximity to tubules (or alternatively the amount of interstitium) was a factor, then the results would not be similar in the mouse and the rat. The rat has approximately threefold fewer 
Decision tree for identifying spermatogonial cell types in the rat testis

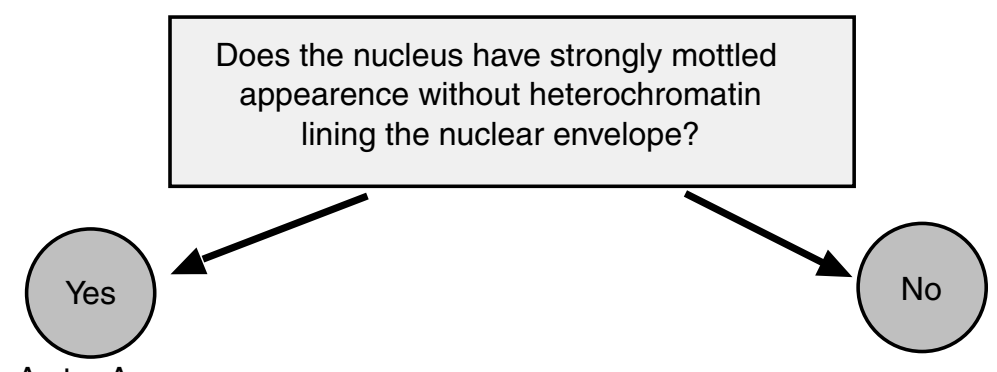
$A_{s}$ to $A_{a l}$
$A_{1}$ to type $B$

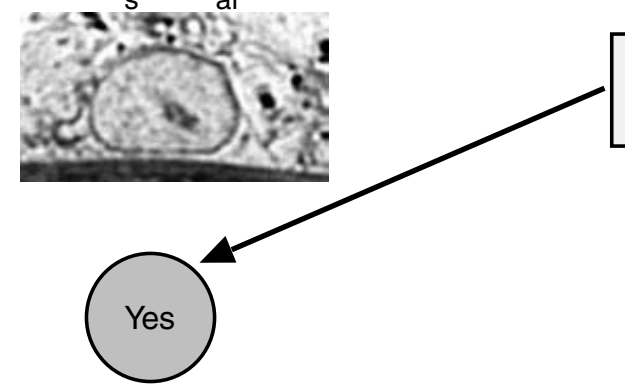

Does the nucleus have virtually no heterochromatin along the nuclear envelope?

$A_{1}$ spermatogonia
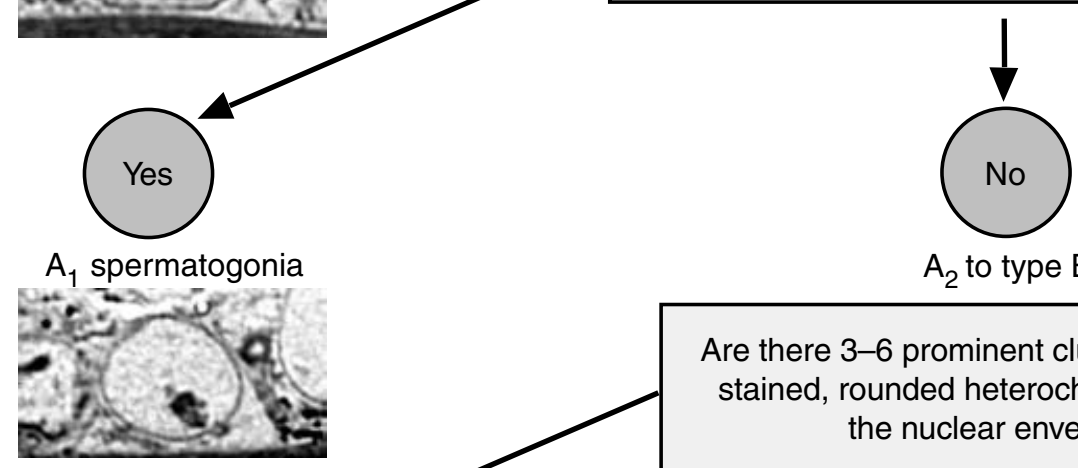

$A_{2}$ to type $B$

Are there 3-6 prominent clumps of densely stained, rounded heterochromatin along the nuclear envelope?
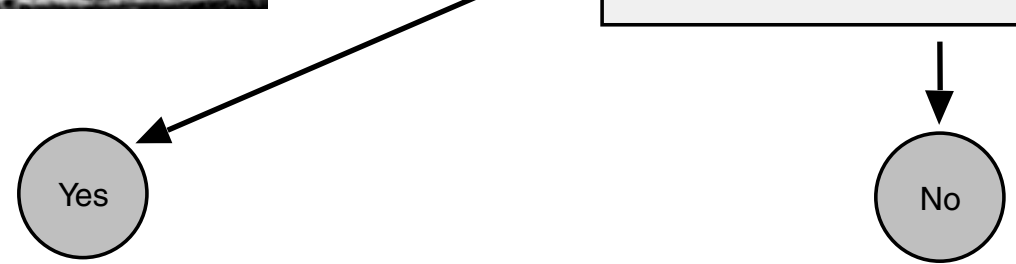

Type B spermatogonia

$A_{2}$ to in spermatogonia

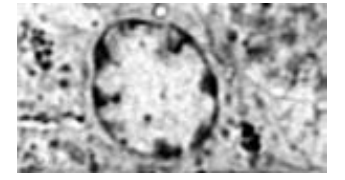

To what extent does heterochromatin rim the nucleus?
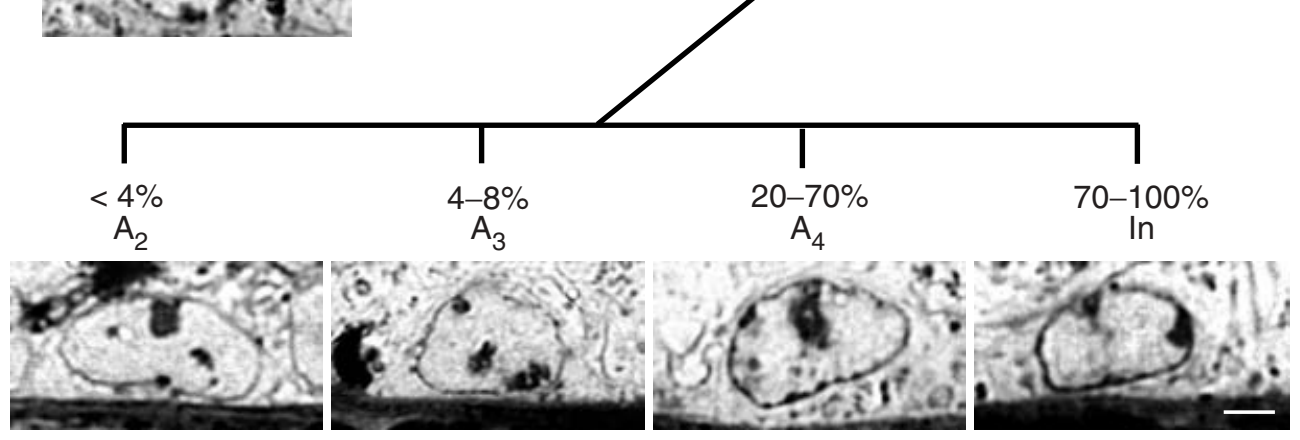

Fig. 4. A yes or no decision tree by which various types of rat spermatogonial cell can be distinguished on the basis of light microscope criteria using the fixation criteria herein specified. Original magnification: $\times 1300$. Scale bars represent $3.6 \mu \mathrm{m}$. 
(a)
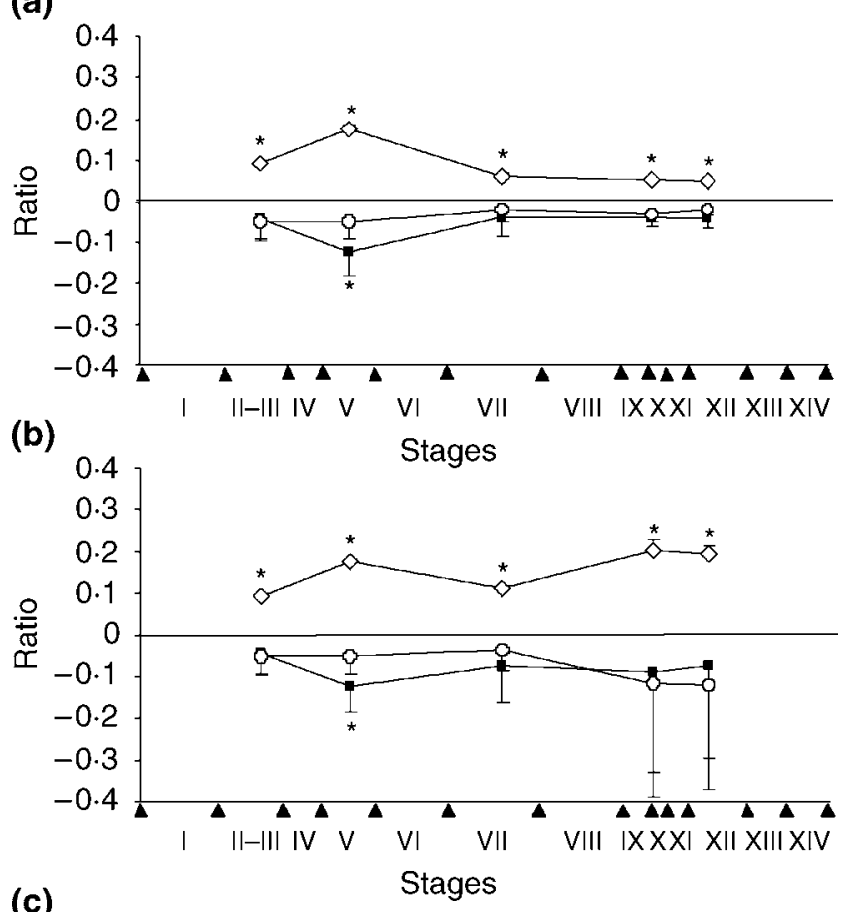

(c)

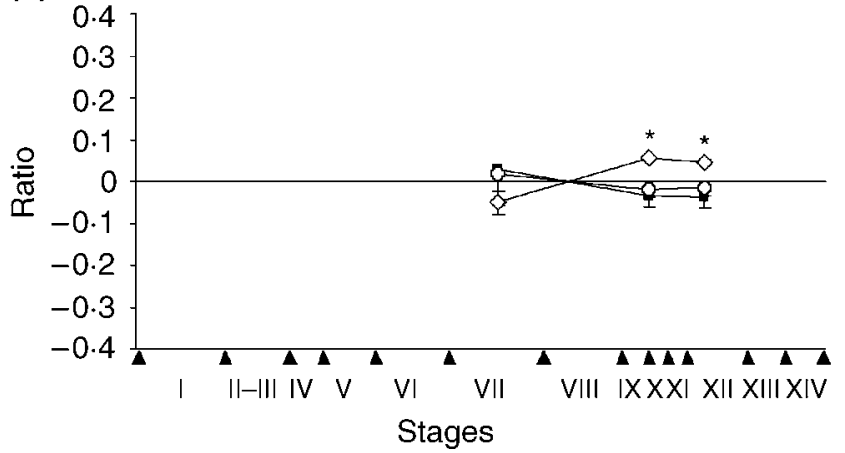

Fig. 5. Linear index graphs showing (a) the topography of all types of spermatogonial cell $\left(A_{\text {single }}-A_{3}\right)$, (b) the topography of the more primitive spermatogonia $\left(A_{\text {single }}, A_{\text {paired }}\right.$ and $\left.A_{\text {aligned }}\right)$ and (c) the distribution of the more advanced spermatogonia $\left(A_{1}-A_{3}\right)$ in rats. Values represent mean \pm SEM. An asterisk over a data point indicates significant non-random distribution $(P<0.05)$. $\diamond$ : interstitial; $\mathbf{a}$ : intermediate; $\bigcirc$ : tubule contact.

tubule-to-tubule contacts than does the mouse, but the adjacent tubules are often very close. The method used in the present study to compensate for the proximity of tubules is represented in the tubule proximity graphs. For example, the Li graph and the proximity graphs of $A_{1}$ to $A_{3}$ cells appear to contradict each other. It is argued that the Li graph shows that tubule contact is not a good measure of spermatogonial preference, but that the proximity graphs show that proximity to tubules (or alternatively the amount of interstitium) is the factor that regulates spermatogonial position. First, it is intuitive that, as spermatogonia continue to divide, their distribution becomes random as they essentially surround the entire seminiferous tubule. Only the proximity graph shows the (a)
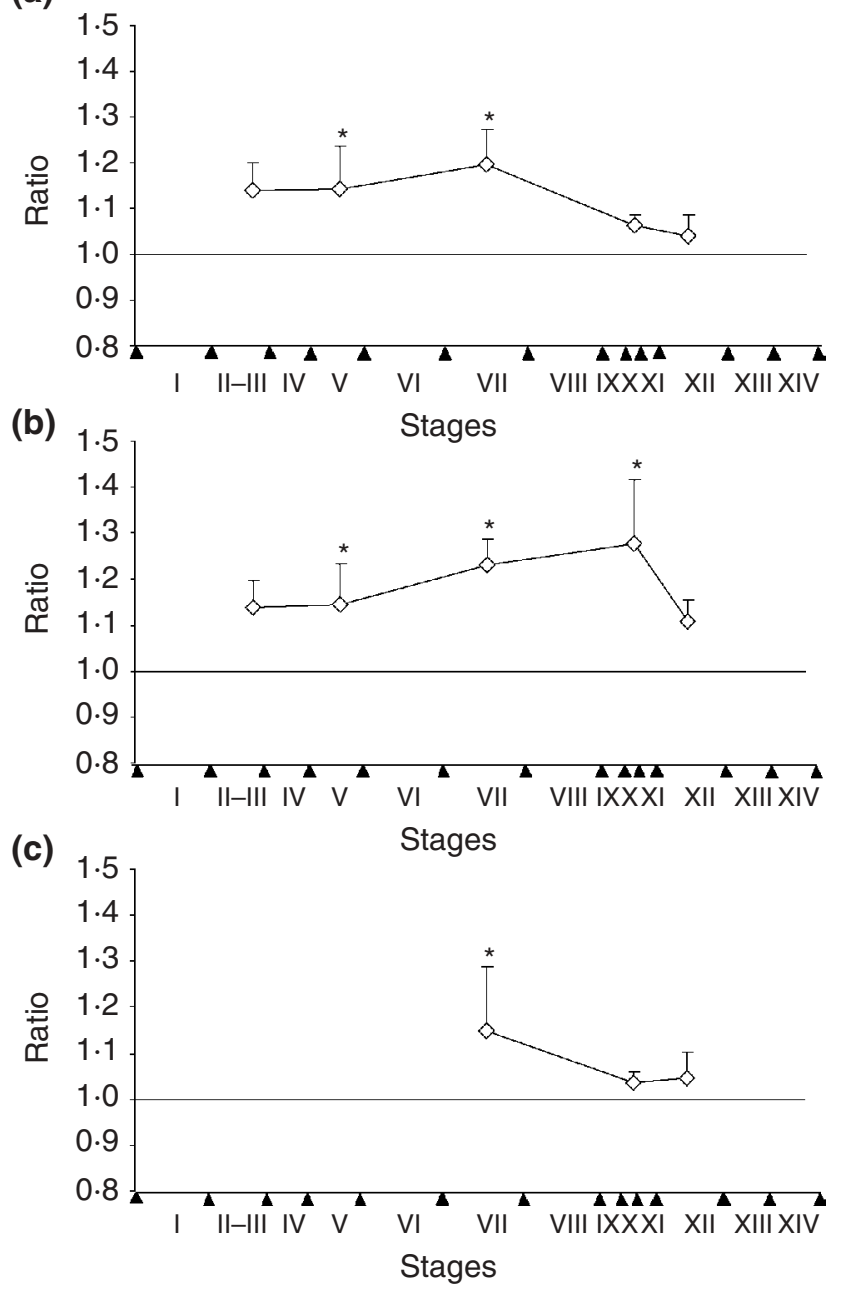

Fig. 6. Tubule proximity graphs showing distribution of (a) all spermatogonia $\left(A_{s}-A_{3}\right)$, (b) primitive spermatogonia $\left(A_{\text {single }}, A_{\text {paired }}\right.$ and $\left.A_{\text {aligned }}\right)$ and $(c)$ advanced spermatogonia $\left(A_{1}-A_{3}\right)$ in rats. Values represent mean $\pm S E M$. Asterisks indicate significant non-random distribution $(P<0.05)$.

random distribution of the more advanced $\left(\mathrm{A}_{2}\right.$ and $\left.\mathrm{A}_{3}\right)$ spermatogonia. This distribution resembles the pattern of spermatogonial distribution in the mouse and takes into account that tubules do not need to make contact to affect spermatogonial position (or, alternatively, to eliminate significant amounts of interstitium), showing that tubule contact per se does not govern spermatogonial position.

It should be noted that the cells associated with a particular position in the seminiferous tubule are the most immature spermatogonia $\left(A_{s}, A_{p r}\right.$ and $\left.A_{a l}\right)$. However, at present this cell grouping cannot be separated on the basis of morphology. As it is a cell group, the data for the entire group reflect the sum of all members of the group. It is possible that some members of the group $\left(A_{s}-\right.$ the stem cell spermatogonia) have a stronger association for the interstitium and other members of the group $\left(A_{p r}\right.$ 


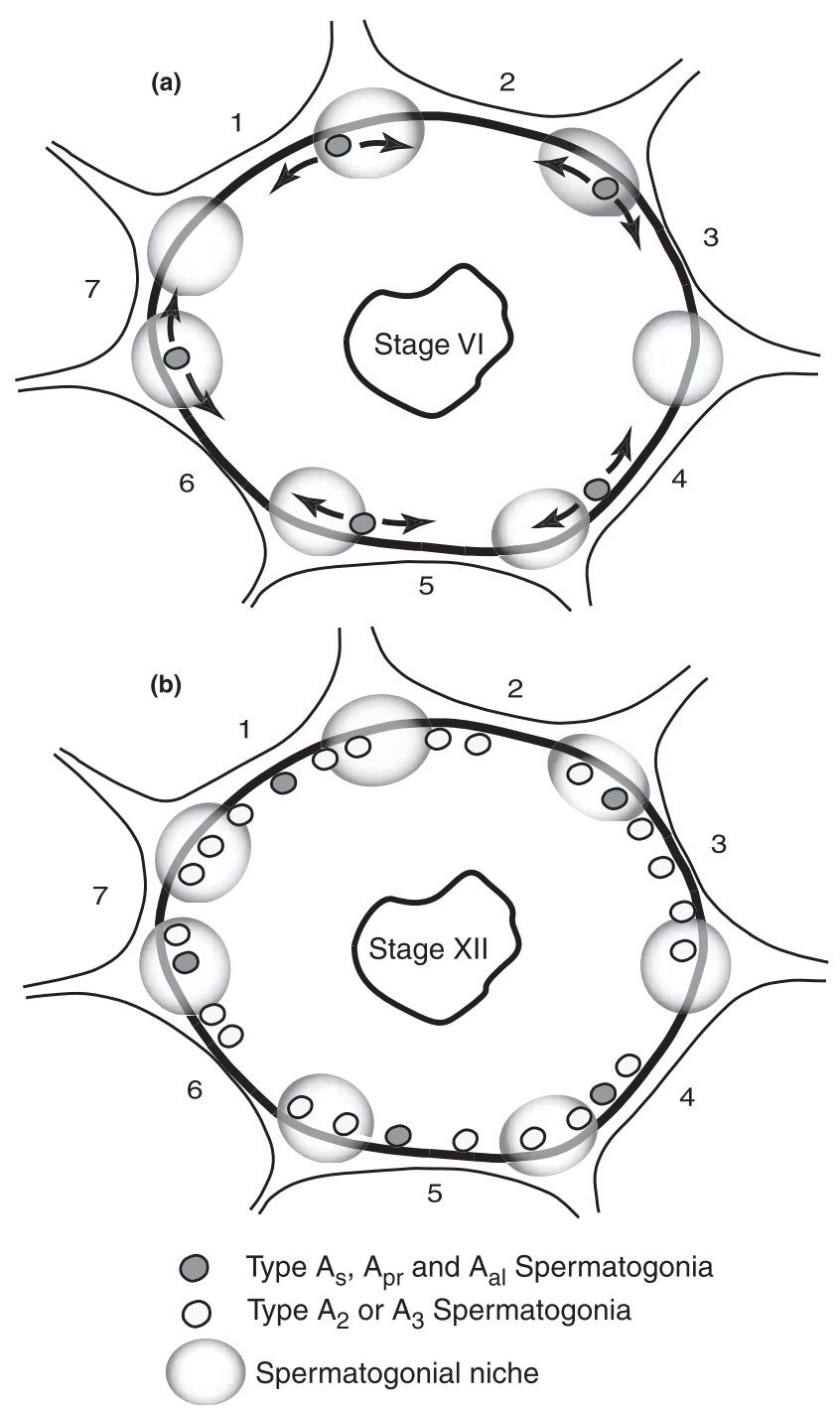

Fig. 7. Drawings of rat seminiferous tubule cross-sections in, illustrating the location of the niches in the interstitial regions of the basal compartment (large circles). (a) In stage $\mathrm{VI}$, the most primitive type $A$ spermatogonia $\left(A_{\text {single }}-A_{\text {aligned }}\right)$ are located preferentially in or close to the interstitial areas. After successive mitoses, the daughter cells spread out laterally from this region (arrows). (b) In stage XII, type $A_{2}$ and $A_{3}$ spermatogonia are distributed randomly in the basal compartment, resulting in the even distribution of spermatogenesis throughout the circumference of the seminiferous tubule. Arabic numbers, adjacent seminiferous tubules.

and $A_{a l}$ ) have a weaker association. The Li graphs and tubule proximity graphs indicate that as there are no significant differences in the position of this primitive group, there is no movement of spermatogonia during the spermatogenic cycle.

The results of a study by Chiarini-Garcia et al. (2001) in mice and the results of the present study strongly indicate that, at least in rodents, the primitive spermatogonia $\left(A_{s}\right.$ up to $\left.A_{a l}\right)$ are topographically positioned in niches. These niches are positioned near the interstitial region, where Leydig cells, macrophages, and lymphatic and blood vessels are concentrated (Fig. 7). These particular locations could represent a strategic position for the niches in which specific factors that may be emanating from the interstitium could control their activity - stimulating or inhibiting. As spermatogenesis is a continuous process of self-renewing cells, the niche is probably important in this process by securing a correct ratio between self-renewal and differentiation of the stem cell daughters to avoid stem depletion or accumulation. It is possible that under the control of the niche, the chance of type $A_{s}$ spermatogonia differentiating is probably very small. However, after division, the $A_{s}$ daughter cells could be pushed away laterally from the niches considering the possible small area of the niche in the basal compartment of the seminiferous tubules. After migrating far from the interstitial area, and with no further effects from the niche, the following $A_{s}$ would then divide becoming type $A_{p r}$ spermatogonia. This could provide a possible regulatory mechanism for the stem cell spermatogonia proliferation. For example, in a symmetrical spermatogonia division (de Rooij and Russell, 2000) a stem cell either divides to form two $A_{s}$ daughter cells (niche-controlled division) or a pair ( $A_{p r}$; no niche-controlled division). Thus, it is postulated that under regulatory mechanisms the niches in the testes could determine the fate and the behaviour of the $A_{s}$ stem spermatogonia. The self-renewing property could be controlled both by cell-autonomous mechanisms and by extrinsic signalling (Morrison et al., 1997); however, further studies are required to determine the regulatory factors that could control the spermatogonial niches. After committed $A_{p r}$ spermatogonia are formed, the spermatogonial divisions ( $A_{p r}$ to type $B$ spermatogonia) and meiotic and spermiogenic phases of the spermatogenesis should develop as reported by Russell et al. (1990) and de Rooij and Russell (2000), with no further effects from the niches.

By definition, a niche is considered a microenvironment in which tissue cells and extracellular matrix can house stem cells and control their self-renewal and progeny production in vivo. They are regulated by signalling molecules and local environmental factors in response to the organism needs when the activity of stem cells is controlled to ensure the correct production of differentiated types of cell (Spradling et al., 2001). In addition, studies in other self-renewing systems have shown that extrinsic signalling has been implicated in controlling stem cell division (Morrison et al., 1997), and in organisms ranging from invertebrates to mammals, this control could be regulated via neighbouring nonmitotic somatic cells (Lin, 1997). As Sertoli cells mediate many aspects of male germ cell development (Russell and Griswold, 1993), this somatic cell would be the natural candidate to participate in the spermatogonial stem cell niches. Recent findings have shown that the major factor in regulating stem cell fate is glial cell 
line derived neutrophic factor (GDNF) produced by Sertoli cells which inhibits stem cell differentiation (Meng et al., 2000). In this regard, GDNF is a good candidate to regulate stem niches. However, it remains to be investigated whether only the Sertoli cells specifically located near spermatogonial stem cells are responsible for GDNF production. A Drosophila gene (piwi) has been characterized which is essential for the division of the stem cell, and not important for the further differentiation of the committed daughter cells (Cox et al., 1998). Recently, a human piwi-like gene called hiwi was identified in the testis. The hiwi gene is correlated with seminomas derived from the malignant proliferation of spermatogonial stem cells or their precursors (Qiao et al., 2002). In this regard, these genes could also be good candidates to regulate spermatogonial stem cell niches.

The present study strongly indicates that spermatogonial niches are present in rats, and that they are located in a specific region of the basal compartment of seminiferous tubules; however, it does not identify the factors that can regulate them. Recent findings indicate that high testosterone concentrations prevent spermatogonial differentiation (Shuttlesworth et al., 2000; Shetty et al., 2001; Tohda et al., 2001). However, low intratesticular testosterone concentrations caused by $\mathrm{GnRH}$-antagonist (leuprolide) markedly increase the transplant efficiency in recipient mice probably due to increased spermatogonial stem cell proliferation (Ogawa et al., 1998; Dobrinski et al., 2001). Once the spermatogonial niches are bordering the interstitial tissues with the testosterone concentrations possibly being highest in such an area, testosterone could have a major role in regulating stem cell niche. However, testosterone can only indirectly affect spermatogonia because they do not possess androgen receptors that in the seminiferous tubules are expressed exclusively in Sertoli and myoid cells (Sar et al., 1990). Once the spermatogonial niches have been identified, the next challenge will be to determine how they are regulated. An interesting approach to reveal the nature of controlling factors would be to investigate the effect of the testosterone concentrations on the spermatogonial niches by applying experimental models in which its concentration is markedly altered.

This work was supported by NIH (HD 35494 to L. D. Russell and a Latin American Fellowship to H. Chiarini-Garcia). H. ChiariniGarcia was also partially supported by the Brazilian Research Foundations CNPq and Fapemig. The technical help of Y. Li (USA), R. Miranda and A. M. Ferreira (Brazil) are gratefully acknowledged.

\section{References}

Bejerknes M and Cheng H (1999) Clonal analysis of mouse intestinal epithelial progenitors Gastroenterology 116 7-14

Brinster RL (2002) Germline stem cell transplantation and transgenesis Science 296 2174-2176
Chiarini-Garcia H and Russell LD (2001) High-resolution light microscopic characterization of mouse spermatogonia Biology of Reproduction $\mathbf{6 5}$ 1170-1178

Chiarini-Garcia H and Russell LD (2002) Characterization of mouse spermatogonia by transmission electron microscopy Reproduction $\mathbf{1 2 3}$ 567-577

Chiarini-Garcia H, Hornick JR, Griswold MD and Russell LD (2001) Distribution of type A spermatogonia in the mouse is not random Biology of Reproduction 65 1179-1185

Cox DN, Chao A, Baker J, Chang L, Qiao D and Lin H (1998) A novel class of evolutionarily conserved genes defined by piwi are essential for stem cell self-renewal Genes and Development 12 3715-3727

Dobrinski I, Ogawa T, Avarbock MR and Brinster RL (2001) Effect of the $\mathrm{Gn}-\mathrm{RH}$-agonist leuprolide on colonization of recipient testes by donor spermatogonial stem cells after transplantation in mice Tissue and Cell 33 200-207

de Rooij DG and Russell LD (2000) All you want to know about spermatogonia but were afraid to ask Journal of Andrology 21 776798

Leblond CP and Clermont Y (1952) Definition of the stages of the cycle of the seminiferous epithelium in the rat Annals of the New York Academy of Sciences 55 548-573

Lin $\mathbf{H}$ (1997) The tao of the stem cells in the germline Annual Reviews of Genetics 31 455-491

Meistrich ML (1998) Hormonal stimulation of the recovery of spermatogenesis following chemo- or radiotherapy Acta Pathologica, Microbiologica et Immunologica Scandinavica 106 37-45

Meng X, Lindahl M, Hyvonen ME et al. (2000) Regulation of cell fate decision of undifferentiated spermatogonia by GDNF Science $\mathbf{2 8 7}$ 1489-1493

Miller SJ, Lavker RM and Sun T-T (1997) Keratinocyte stem cells of cornea, skin and hair follicules. In Stem Cells pp 331-362 Ed. CS Potten. Academic Press, London

Morrison SJ, Shah NM and Anderson DJ (1997) Regulatory mechanisms in stem cell biology Cell $\mathbf{8 8} 287-298$

Ogawa T, Dobrinski I, Avarbock MR and Brinster R (1998) Leoprolide, a gonadotropin-releasing hormone agonist, enhances colonization after spermatogonial transplantation into mouse testes Tissue and Cell $\mathbf{3 0}$ 583-588

Parreira GG, Ogawa T, Avarbock MR, Franca LR, Brinster RL and Russell LD (1998) Development of germ cell transplants in mice Biology of Reproduction 59 1360-1370

Qiao D, Zeeman A-M, Deng W, Looijenga LHJ and Lin H (2002) Molecular characterization of hiwi, a human member of the piwi gene family whose overexpression is correlated to seminomas Oncogene 21 39883999

Russell LD and Burguet S (1977) Ultrastructure of Leydig cells as revealed by secondary tissue treatment with ferrocyanide-osmium mixture Tissue and Cell 9 751-766

Russell LD and Griswold MD (1993) The Sertoli Cell Cache River Press, Vienna

Russell LD, Ettlin RA, Sinha Hikim AP and Clegg ED (1990) Histological Histopathological Evaluation of the Testis Cache River Press, Vienna

Sar M, Lubahn DB, French FS and Wilson EM (1990) Immunohistochemical localization of the androgen receptor in rat and human tissues Endocrinology 127 3180-3186

Shetty G, Wilson G, Huhtaniemi I, Boettger-Tong H and Meistrich ML (2001) Testosterone inhibits spermatogonial differentiation in juvenile spermatogonial depletion mice Endocrinology 142 2789-2795

Shuttlesworth GA, de Rooij DG, Huhtaniemi I, Reissmann T, Russell LD, Shetty G, Wilson G and Meistrich ML (2000) Enhancement of A spermatogonial proliferation and differentiation in irradiated rat by GnRF antagonist administration Endocrinology 141 37-49

Spangrude GS, Heimfeld S and Weissman IL (1988) Purification and characterization of mouse hematopoietic stem cells Science 241 58-62

Spradling A, Drummond-Barbosa D and Kai T (2001) Stem cells find their niche Nature 414 98-104

Sprando RL (1990) Perfusion of the rat testis through the heart using heparin. In Histological Histopathological Evaluation of the Testis pp 277-280 
Eds LD Russell, RA Ettlin, AP Sinha Hikim and ED Clegg. Cache River Press, Vienna

Strauss RE (1979) Reliability estimates for Ivlev's electivity index, the forage ratio, and a proposed linear index of food selection Transactions of the American Fisheries Society 108 344-352

Tohda A, Matsumiya K, Tadokoro Y, Yomogida K, Miyagawa Y, Dohmae K, Okuyama A and Nishimune Y (2001) Testosterone suppress spermatogenesis in juvenile spermatogonial depletion (jsd) mice Biology of Reproduction 65 532-537

van den Aardweg GJ, de Ruiter-Bootsma AL, Kramer MF and Davids JAG (1982) Growth of spermatogenetic colonies in the mouse testis after irradiation with fission neutrons Radiation Research 89 150-165 van Pelt AMM and de Rooij DG (1990a) The origin of the synchronization of the seminiferous epithelium in vitamin A-deficient rats after vitamin A replacement Biology of Reproduction 42 677-682

van Pelt AMM and de Rooij DG (1990b) Synchronization of the seminiferous epithelium after vitamin A replacement in vitamin A-deficient mice Biology of Reproduction 43 363-367

Received 24 February 2003.

First decision 22 May 2003.

Revised manuscript received 1 July 2003.

Accepted 24 July 2003. 